\title{
Original
}

\section{Análisis de diez años de registro de malformaciones congénitas en Costa Rica}

\author{
(Ten year analysis of the national registry of congenital \\ anomalies in Costa Rica)
}

María de la Paz Barboza-Argüello, Lila María Umaña-Solís

\section{Resumen}

Centro de Registro de Enfermedades Congénitas (CREC), Instituto Costarricense de Investigación y Enseñanza en Nutrición y Salud (INCIENSA)

Abreviaturas: CREC, Centro de Registro de Enfermedades Congénitas; CCP, Centro Centroamericano de Población; CCSS, Caja Costarricense de Seguro Social; CIE 10, Décima Clasificación Internacional de Enfermedades; IC, Intervalo de confianza; INCIENSA, Instituto Costarricense de Investigación y Enseñanza en Nutrición y Salud; INEC, Instituto Nacional de Estadística Y Censos; OMS, Organización Mundial de la Salud; OPS, Organización Panamericana de la Salud; OR, Odds Ratio; RN, recién nacido; $\mathrm{PH}$, paladar hendido; RP, razón de prevalencia; Sd, síndrome; SPSS, Statistical Package for Social Sciences.

Correspondencia:

María de la Paz Barboza Argüello.

E.mail:mbarboza@inciensa.sa.cr
Justificación y objetivo: Las malformaciones congénitas constituyen la segunda causa de mortalidad infantil en Costa Rica; este estudio se realizó con el objetivo de determinar la prevalencia de nacidos malformados y polimalformados durante 1996-2005, y describir su comportamiento en tiempo, lugar y persona.

Métodos: Se consideró malformado simple a todo individuo cuya malformación se hubiera codificado mediante la CIE 10, y polimalformado a los recién nacidos diagnosticados con un síndrome o con al menos 2 malformaciones codificadas. Fuente de información: Centro de Registro de Malformaciones Congénitas, (base poblacional) y Centro Centroamericano de Población (datos en línea). Se analizaron las prevalencias en tiempo, según condición al nacer, edad de la madre, provincia de residencia y sexo del recién nacido. Se realizó además una regresión logística, tomando como variable dependiente la presencia o no de malformación, y como independiente la edad y residencia de la madre, así como el sexo del recién nacido.

Resultados: Se registraron 11.099 recién nacidos malformados simples, prevalencia del 1,47\% (IC $95 \%=1,44-1,49$ ), y 2.629 polimalformados, prevalencia de 34,8/10.000 nacidos (IC 95\% $=33,4-36,1)$. Dentro del grupo de polimalformados, 1.065/2.629 (40,5\%) presentaron síndromes y 1.564/2.629 (59,5\%) evidencian asociación de dos o más malformaciones. Las malformaciones más frecuentes fueron aquellas del sistema osteomuscular. El síndrome más frecuente fue el de Down. Durante el segundo quinquenio analizado se presentó un aumento significativo de la prevalencia de polimalformados en la provincia de Limón. La prevalencia de malformados y polimalformados fue significativamente mayor tanto en el sexo masculino como para las madres de más de 35 años. El riesgo en mujeres con edades superiores a 35 años, de dar a luz un polimalformado, fue 2,4 veces el de aquellas de menor edad (IC 95\%=2,2-2,6).

Conclusión: La edad de la madre y el sexo del recién nacido son factores de riesgo para la presencia de malformaciones. Se requiere de estudios analíticos para determinar las causas del aumento significativo de la prevalencia de polimalformados, a partir de 2001 en la provincia de Limón.

Descriptores: polimalformado, registro, malformaciones congénitas, Costa Rica

\section{Abstract}

Background and aim: Congenital malformations are the second leading cause of infant mortality in Costa Rica. We studied the prevalence of malformed and polymalformed newborns between the years of 1996 and 2005 and describe the secular trend of these congenital diseases. 
ISSN 0001-6002/2008/50/4/221-229 Acta Médica Costarricense, (C2008 Colegio de Médicos y Cirujanos
Methods: The population based passive registry of the Congenital Malformations Registry Center, INCIENSA and the online database of the Central American Center for Population of the University of Costa Rica were the data sources. A newborn with malformation coded with a single CIE 10 number was classified as "simple" and as "polymalformed" when he or she presented at least 2 malformations or a specific syndrome. Prevalence of these congenital diseases were analyzed in time according to birth's condition (dead or alive), mother's age, province of residence, and newborn's gender. Additionally, a logistic regression model was performed using the presence or absence of malformation as dependent variable and the mother's age, place of residence and newborn gender as independent variables.

Results: Of the total of newborns during the study period ( $\mathrm{n}=755978), 1.47 \%(95 \%=1.44-$ 1.49) were malformed (11 099 newborns) and $34.8 / 10000$ infants $(95 \%=33.4$ to 36.1$)$ were polymalformed (2 629 newborns).

Of the total malformed newborns, $40.5 \%$ presented syndromes and $59.5 \%$ had association of 2 or more malformations. Malformations related to the skeletalmuscle system and to Down's syndrome were the most common. Different from findings in other provinces, the prevalence of polymalformations increased significatively in the Province of Limon during the second quinquennium of the study period. The prevalence of malformed and polymalformed newborns was significantly higher in males as well as in children born of mothers older than 35 years. The risk of having a polymalformed newborn baby was 2.4 times higher in women aged $\geq 35$ years than in those with lower age $(95 \%=2.2-2.6)$.

Conclusion: This study suggests that mother's age and newborn's gender confer an important risk for delivering children with malformations. More research is needed to achieve a comprehensive understanding of the increased prevalence of multiple malformations in the Province of Limon since 2001.

Key words: polymalformed, registration, birth defects, Costa Rica

Recibido: 29 de febrero de $2008 \quad$ Aceptado: 15 de julio de 2008
Las malformaciones congénitas constituyen un grupo de enfermedades de alta mortalidad, cuyo manejo y rehabilitación no siempre es exitoso. Muchas de ellas son de evolución crónica y pueden producir secuelas que representan una desventaja social, con un alto costo para la familia y el Estado. ${ }^{1}$

En la actualidad, los defectos congénitos constituyen la causa principal de mortalidad infantil en Estados Unidos y en la mayoría de los países desarrollados. ${ }^{2}$ En Costa Rica las anomalías congénitas constituyen la segunda causa de mortalidad infantil, superadas únicamente por las afecciones perinatales. ${ }^{3,4}$ Para 2004, con la mortalidad infantil más baja de los últimos 55 años $(9,25$ por mil nacidos vivos), las malformaciones congénitas en Costa Rica ocuparon el segundo lugar, constituyendo el $30,1 \%$ de las muertes en menores de 1 año. En 2002, las malformaciones congénitas múltiples, no clasificadas, constituyeron la novena causa de muerte infantil en Costa Rica. ${ }^{5}$ Las malformaciones congénitas constituyeron la primera causa de muerte en el Hospital Nacional de Niños "Dr. Carlos Sáenz Herrera" y la cuarta causa de egreso hospitalario, durante el año 2007, según se reporta en el informe estadístico de ese año, de dicho hospital.
La OMS define defecto congénito como: "toda anomalía del desarrollo morfológico, estructural, funcional o molecular, presente al nacer (aunque se manifieste después del nacimiento". Los defectos congénitos abarcan una amplia variedad de patologías; su prevalencia puede llegar hasta un $7-10 \%$ durante el primer año de vida.

Si se tiene en cuenta que los defectos congénitos en general contribuyen de manera importante en las discapacidades del desarrollo; ${ }^{6}$ que aproximadamente entre el $20 \%$ y el $40 \%$ de las muertes ocurridas en el primer año de vida se deben a esas malformaciones; que una gran cantidad de especialistas se verán implicados en su diagnóstico y tratamiento y que la mayoría de los pacientes requerirá tratamiento de por vida, o bien que su tratamiento puede dejar secuelas, se constata que estamos frente a un problema de salud pública de primer orden.

Existen muchas formas de clasificar los defectos congénitos; según el número de ellos presentes en el recién nacido observar 2 grupos de niños malformados: los que presentan un único defecto (defectos aislados) y los que tienen más de uno (polimalformados). ${ }^{7}$ En este último grupo se reconocen, a su vez, 3 tipos de niños afectados: 
1. Aquellos cuyos defectos correspondan con alguno de los síndromes descritos en la bibliografía. Los síndromes generalmente suelen tener la misma causa y el mismo riesgo familiar.

2. Niños con defectos congénitos que afectan a estructuras corporales diferentes aparentemente no relacionadas, en quienes no se ha podido diagnosticar ningún síndrome conocido y no se ha identificado un agente causal.

3. Niños con un único defecto del desarrollo, pero en quienes, ese defecto, ha dado lugar secundaria y secuencialmente, a alteraciones de otras estructuras cuyo desarrollo morfológico era normal. Las secuencias pueden ser producidas por muchos agentes causales, pueden manifestarse en polimalformados sin etiología establecida, como síndromes o como una secuencia aislada.

Desde 1985, mediante el decreto ejecutivo $16488-\mathrm{S},{ }^{9}$ se estableció en Costa Rica, el Centro de Registro de Enfermedades Congénitas (CREC), el cual, establece la obligatoriedad de notificación de las malformaciones congénitas detectadas al nacimiento. ${ }^{9}$ En el país se registran todas las malformaciones observadas en un recién nacido, sin límite de número, y se codifican según la Clasificación Internacional de Enfermedades (CIE 10). ${ }^{10}$ El CREC realiza informes y publicaciones periódicas a instituciones nacionales e internacionales con base en la información recolectada, sin embargo, a la fecha no se ha analizado la información correspondiente al recién nacido malformado múltiple. La dificultad de monitorear a los recién nacidos polimalformados radica en la gran cantidad de definiciones y clasificaciones que existen y la forma como se almacena actualmente la información.

En Costa Rica, no se conoce la prevalencia de malformados múltiples ni el porcentaje que representan de todos los malformados. El objetivo del presente estudio consiste en determinar la prevalencia de recién nacidos con malformaciones múltiples en Costa Rica durante el período 1996-2005 y describir su comportamiento a través del tiempo, distribución geográfica y características personales como edad de la madre, sexo del recién nacido y condición al nacimiento.

\section{Materiales y métodos}

La fuente de información fue el Centro de Registro de Enfermedades Congénitas, que realizó la captura de información mediante la boleta de notificación obligatoria del CREC. La información se encuentra almacenada en archivos físicos y en forma electrónica.

El Registro se alimenta con la información que envían los 25 hospitales con servicio materno-infantil de la CCSS y algunos hospitales privados. Se estima que su cobertura es mayor del 95\% de los nacimientos del país.
El Registro es parte de los informes de declaración obligatoria que se reciben en el Ministerio de Salud, según normas establecidas. Es pasivo y de base poblacional. ${ }^{11}$

En el Registro se incluyen todos los recién nacidos malformados, vivos u óbitos de 500 gramos de peso o más, nacidos en maternidades de los centros de salud. No se consideran los nacimientos ocurridos fuera de las maternidades, a ser que se incluyan en el reporte diario del hospital para registro estadístico. ${ }^{12}$ Según las normas de operación, todos los recién nacidos, vivos y óbitos, se examinan clínicamente en busca de malformaciones; la observación se extiende hasta su alta en la maternidad.

$\mathrm{La}$ información correspondiente a los nacimientos durante el período de estudio se extrajo de la base de datos en línea del Centro Centroamericano de Población (CCP).

El análisis se realizó con los datos registrados a partir de 1996, debido a que mediante análisis previos del Registro publicados en el libro "Prevalencia de enfermedades congénitas por provincias y cantones, Costa Rica 19872000" 11 , se logró identificar que es a partir de este año cuando no solo mejora la cobertura del Registro, sino además, la calidad de su información.

No se tomaron en cuenta para este trabajo variables que identificaran a las madres o a los recién nacidos por lo que nunca se comprometió la confidencialidad. El estudio fue aprobado por el Comité Ético Científico del INCIENSA.

Se consideró malformado simple a todo individuo cuyas malformaciones fueron codificadas con un único número de la CIE 10 y cuyo diagnóstico correspondiera a una malformación. Aquellos con diagnósticos CIE 10 que incluyen más de una malformación (como los síndromes) fueron considerados como polimalformados. Por lo tanto, el total de recién nacidos polimalformados estuvo constituido por la suma de los recién nacidos con síndromes identificados en el período de estudio, más los recién nacidos con al menos 2 malformaciones registradas en dicho período.

Debido a que en el Registro de malformaciones se incluye los recién nacidos vivos y los óbitos, se tomó como denominador para el cálculo de la prevalencia a los nacimientos durante el período en estudio, más las defunciones fetales presentadas en ese período.

Ante la eventualidad de que existiera un subregistro de los óbitos, se realizó además del cálculo de la prevalencia para los recién nacidos malformados vivos, la prevalencia de los óbitos malformados, tomando como denominador las defunciones fetales. Se compararon las prevalencias encontradas en vivos y óbitos, para lo que se calcularon intervalos de confianza al $95 \% .{ }^{13}$

Se analizaron las prevalencias tomando en cuenta el año de registro, la provincia de residencia de la madre, la edad de la madre y el sexo del recién nacido. Se analizó cada una de 
estas variables de forma aislada y se realizó también un análisis de regresión logística no condicional, tomando como variable dependiente la presencia o no de malformación, y como variables independientes la edad de la madre, el sexo del recién nacido y la provincia de residencia de la madre.

Logit $P($ malformado $)=\alpha+\beta 1$ edad madre $+\beta 2$ sexo recién nacido $+\beta 3$ provincia residencia madre.

\section{Resultados}

Durante el período en estudio, de 1996 a 2005, se registraron 11.099 recién nacidos con malformaciones; hubo 750.706 nacimientos en total y 5.272 defunciones fetales (óbitos), con una prevalencia del 1,45\% ó 145 recién nacidos malformados por cada 10000 nacidos, y un 4,06\% ó 406 óbitos malformados, por cada 10000 defunciones fetales. La prevalencia total de recién nacidos malformados, independientemente de la condición al nacimiento, fue del 1,47\% ó 147/10000 nacidos (Cuadro 1).

El 76,3\% de los nacidos malformados presentaron malformaciones simples, y el $23,7 \%$ malformaciones múltiples; el 40,5\% de los polimalformados correspondió a síndromes clasificados según la CIE10, y el 59,5\% se presentó como asociación de 2 ó más malformaciones (Figura 1).

Al analizar la condición al nacimiento y el hecho de ser malformado simple o múltiple, como puede observarse en el Cuadro 2, la prevalencia de fallecidos en los polimalformados es 5,48 veces mayor que la de los nacidos vivos. Esta razón de prevalencia es significativamente mayor que la que se presenta en los malformados simples y en los malformados en general.

A pesar de que la prevalencia de malformaciones en óbitos es mayor, al tomar en cuenta toda la población (vivos y fallecidos), la prevalencia encontrada en los malformados (146,8 por 10000), no es diferente a la que se presenta en los nacidos vivos $(144,8$ por 10000$)$ debido a que se traslapan intervalos de confianza. Ello se debe probablemente a que las defunciones fetales apenas representan un $0,7 \%$ de todos los nacimientos.

El grupo de malformaciones más frecuentes fueron las del sistema osteomuscular, seguido por el grupo de ojo, oído, cara y cuello (Cuadro 3). Los síndromes que se diagnosticaron con mayor frecuencia fueron de Down, Edwards y Pateau (Cuadro 4).

Al revisar el comportamiento de las malformaciones a través del tiempo, se observa que en 2001 se presentó un aumento, tanto en las malformaciones simples como en los polimalformados (Figura 2).

Al analizar el porcentaje de polimalformados por provincia y año de nacimiento, no se encontraron diferencias significativas en el tiempo ni en la provincia de residencia de la madre (Cuadro 5). Sin embargo, al estratificar la prevalencia de polimalformados por provincia de residencia, se observó que durante el segundo quinquenio analizado, 2001-2005 (Cuadro 6), la prevalencia de polimalformados en Limón aumentó significativamente. Al comparar la prevalencia de las provincias con la de polimalformados en el nivel nacional, Alajuela y Guanacaste presentaron una prevalencia menor a la nacional.

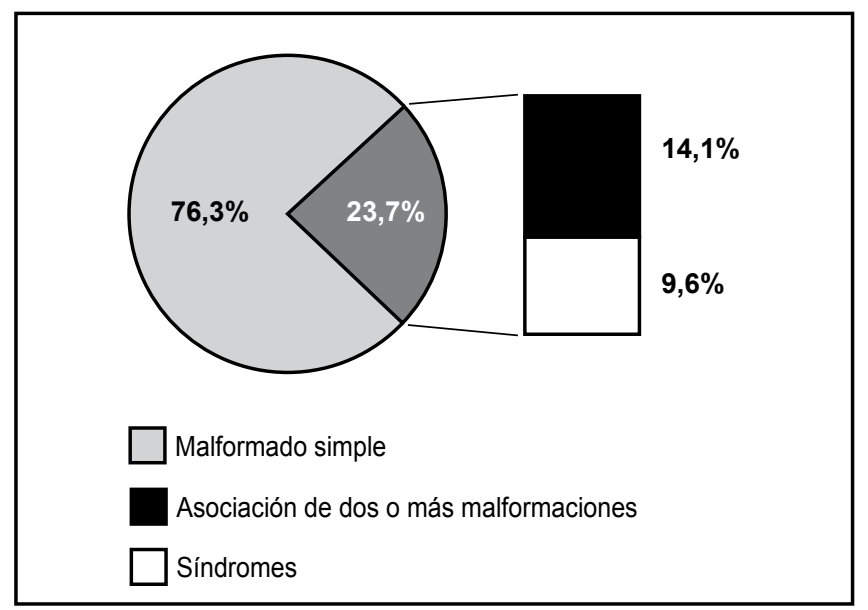

Figura 1. Distribución de recién nacidos malformados, según número de malformaciones y tipo de polimalformado, Costa Rica, 1996-2005.

\begin{tabular}{|c|c|c|c|c|c|c|}
\hline \multicolumn{7}{|c|}{$\begin{array}{l}\text { Cuadro } 1 \text { Prevalencia de recién nacidos con malformaciones congénitas según condición al } \\
\text { nacimiento, Costa Rica, 1996-2005 }\end{array}$} \\
\hline \multirow[b]{2}{*}{ Tipo de malformado } & \multicolumn{2}{|r|}{ Vivos } & \multicolumn{2}{|r|}{ Óbitos } & \multicolumn{2}{|r|}{ Total } \\
\hline & $\begin{array}{l}\text { Casos } \\
96-05\end{array}$ & $\begin{array}{l}\text { Prevalencia* } \\
\text { (IC 95\%) }\end{array}$ & $\begin{array}{l}\text { Casos } \\
96-05\end{array}$ & $\begin{array}{l}\text { Prevalencia * } \\
\text { (IC 95\%) }\end{array}$ & $\begin{array}{l}\text { Casos } \\
96-05\end{array}$ & $\begin{array}{l}\text { Prevalencia* } \\
\text { (IC 95\%) }\end{array}$ \\
\hline Malformado simple & 8349 & $111(108-113)$ & 117 & 222(182 -261.7) & 8470 & $112(110.9-114)$ \\
\hline Polimalformado & 2526 & $33.6(32-34.9)$ & 97 & 184(147.7-220) & 2629 & $34.8(33.4-36)$ \\
\hline Total RN malformados & 10875 & $144.8(142-147)$ & 214 & 406(352.6-459) & 11099 & $146.8(144-149)$ \\
\hline
\end{tabular}

* Prevalencia por 10000

Fuente: CREC y CCP 


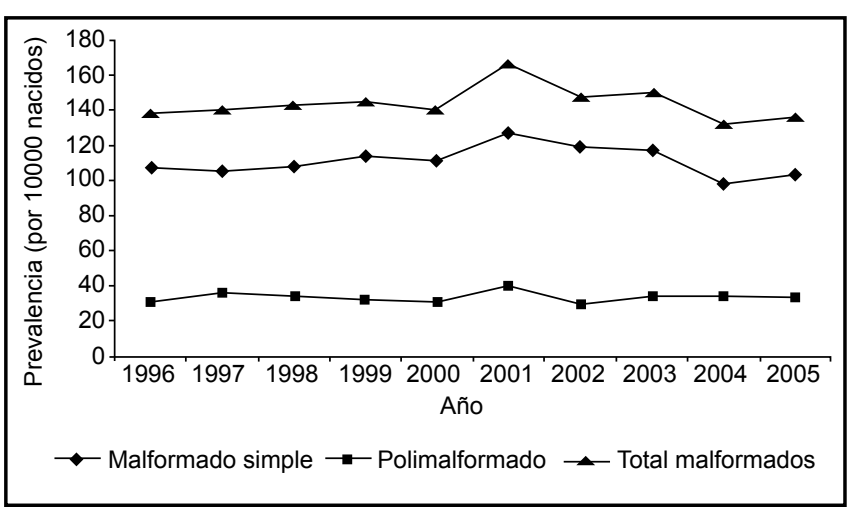

Figura 2. Prevalencia de recién nacidos vivos con malformaciones congénitas simples y múltiples, según año de nacimiento (prevalencia por 10 000), Costa Rica 1996-2005.

Al analizar la información tomando en cuenta la edad de la madre, se encontró que la prevalencia de malformaciones en los hijos de las mayores de 35 años es significativamente mayor a la de las mujeres en edad fértil y en menores de 20 años; la razón de prevalencia encontrada es significativamente mayor en los recién nacidos con malformaciones múltiples, como se observa en los Cuadros 7, 8, es decir, que una mujer de 35 años o más tiene mayor riesgo de parir un recién nacido malformado múltiple.

Aunque la prevalencia de malformaciones simples fue levemente mayor en recién nacidos masculinos, esta no es significativa (se traslapan intervalos de confianza). Sin embargo, al considerar el total de recién nacidos, se observa que la prevalencia es significativamente mayor en el sexo masculino. Esta diferencia se encuentra determinada por los malformados múltiples (Cuadro 9).

Los resultados de la regresión logística se resumen en el cuadro 10, es importante aclarar que las interacciones entre

\begin{tabular}{|lcc|}
\hline $\begin{array}{l}\text { Cuadro 2. Razón de prevalencia (óbitos / vivos) } \\
\text { de recién nacidos con malformaciones, según } \\
\text { condición al nacer, }\end{array}$ & Costa Rica, & 1996-2005 \\
\hline Tipo de malformación & R.P & $($ IC 95\%) \\
\hline Simple & 1.99 & $(1.66-2.4)$ \\
Polimalformado & 5.48 & $(4.5-6.7)$ \\
Total & 2.8 & $(2.4-3.2)$ \\
\hline
\end{tabular}

Fuente: CREC y CCP

las variables independientes no fueron significativas, pero sí hay diferencias de OR entre los estratos de edad y provincia; el estadístico de Wald presentó valores de $p<0,0001$, para ambas variables (grupo de edad y provincia de residencia de la madre).

En las madres con edad de 35 años o más el OR es de 1,35 con un valor de $\mathrm{p}<0,001$. Al analizar la variable "provincia", tomando como base la de San José, se observa que las de Alajuela y Guanacaste tienen OR menores a 1; en las demás provincias se obtuvieron OR mayores a 1. Estos resultados concuerdan con los obtenidos del análisis bivariado (Cuadro 8).

\section{Discusión}

Se estima que mundialmente, la prevalencia de malformaciones oscila entre el $1,5-4 \% .{ }^{14}$ Este porcentaje puede incrementarse hasta un $7 \%$ durante el primer año de vida, ${ }^{6}$ lo que se debe a que algunas de las malformaciones

\section{Cuadro 3. Prevalencia de malformaciones congénitas por grupo de malformación, según CIE 10, Costa Rica, 1996-2005}

\begin{tabular}{|lcc|}
\hline Grupo de malformación congénita (CIE 10) & $\begin{array}{l}\text { Prevalencia } \\
\text { por 10000 }\end{array}$ & IC 95\% \\
\hline Malformaciones y deformaciones congénitas del sistema osteomuscular (Q65 - Q79) & 69,8 & $(67,9-71,6)$ \\
Malformaciones congénitas del ojo, del oído, de la cara y del cuello (Q10 - Q18) & 22,9 & $(21,8-23,9)$ \\
Malformaciones congénitas de los órganos genitales (Q50 - Q56) & 16,2 & $(15,3-17,1)$ \\
Malformaciones congénitas del sistema circulatorio (Q20 - Q28) & 15,1 & $(14,2-15,9)$ \\
Malformaciones congénitas del sistema nervioso (Q00 - Q07) & 13,2 & $(12,4-14,0)$ \\
Otras malformaciones congénitas (Q80 - Q89) & 11,9 & $(11,1-12,7)$ \\
Anomalías cromosómicas no clasificadas en otra parte (Q90 - Q99) & 11,7 & $(10,9-12,5)$ \\
Otras malformaciones del sistema digestivo (Q38 - Q45) & 11,4 & $(10,6-12,2)$ \\
Fisura del paladar y labio leporino (Q35 - Q37) & 9,3 & $(8,6-10)$ \\
Malformaciones congénitas del sistema urinario (Q60 - Q64) & 3,2 & $(2,8-3,6)$ \\
Malformaciones congénitas del sistema respiratorio (Q30 - Q34) & 1,7 & $(1,4-1,9)$ \\
\hline
\end{tabular}




\section{Cuadro 4. Prevalencia de los principales síndromes diagnosticados, según condición al nacimiento,}

y razón de prevalencia entre óbitos y vivos (Prevalencia por 10000 ), Costa Rica 1996-2005

\begin{tabular}{|c|c|c|c|c|c|c|c|c|}
\hline CIE10 & Síndrome & $\begin{array}{l}\text { Casos } \\
\text { (total) }\end{array}$ & Prevalencia & $\begin{array}{l}\text { Casos } \\
\text { (vivos) }\end{array}$ & Prevalencia $^{a}$ & $\begin{array}{l}\text { Casos } \\
\text { (óbitos) }\end{array}$ & Prevalencia $^{\mathrm{b}}$ & $\begin{array}{l}\text { R.P. } \\
(\mathrm{o} / \mathrm{v})\end{array}$ \\
\hline $\begin{array}{l}\text { Q902 y } \\
\text { Q909 }\end{array}$ & Síndrome de Down & 703 & 9,30 & 694 & 9,24 & 9 & 17,07 & 1,30 \\
\hline Q917 & Síndrome de Patau & 94 & 1,24 & 87 & 1,16 & 7 & 13,28 & 8,05 \\
\hline $\begin{array}{l}\text { Q910y } \\
\text { Q913 }\end{array}$ & Síndrome de Edwards & 69 & 0,91 & 67 & 0,89 & 2 & 3,79 & 2,99 \\
\hline $\begin{array}{l}\text { Q771 } \\
\text { Q772 y } \\
\text { Q774 }\end{array}$ & $\begin{array}{l}\text { Osteocondrodisplasias } \\
\text { (acondroplasia, enanismo } \\
\text { tanatoforico) }\end{array}$ & 47 & 0,62 & 43 & 0,57 & 4 & 7,59 & 9,30 \\
\hline Q897 & $\begin{array}{l}\text { Malformaciones congénitas } \\
\text { múltiples no clasificadas en } \\
\text { otra parte }\end{array}$ & 42 & 0,56 & 32 & 0,43 & 10 & 18,97 & 31,25 \\
\hline Q794 & $\begin{array}{l}\text { Sd de Prune Belly o } \\
\text { abdomen en ciruela pasa }\end{array}$ & 22 & 0,29 & 22 & 0,29 & 0 & & \\
\hline Q969 & $\begin{array}{l}\text { Síndrome de Turner } \\
\text { Sd de malformaciones } \\
\text { congénitas que afectan }\end{array}$ & 21 & 0,28 & 21 & 0,28 & 0 & - & - \\
\hline Q870 & $\begin{array}{l}\text { principalmente la apariencia } \\
\text { facial }\end{array}$ & 17 & 0,22 & 16 & 0,21 & 1 & 1,90 & 6,25 \\
\hline Q606 & Síndrome de Potter & 13 & 0,17 & 13 & 0,17 & 0 & 0,00 & 0,00 \\
\hline Q872 & $\begin{array}{l}\text { Sd de malformaciones } \\
\text { congénitas que afectan } \\
\text { principalmente los miembros }\end{array}$ & 9 & 0,1 & 7 & 0,09 & 2 & 3,79 & 28,57 \\
\hline $\begin{array}{l}\text { Q780 y } \\
\text { Q783 }\end{array}$ & $\begin{array}{l}\text { Osteogénesis imperfecta y } \\
\text { displasia diafisiaria }\end{array}$ & 9 & 0,12 & 9 & 0,12 & 0 & - & - \\
\hline Q86 & $\begin{array}{l}\text { Sd de malformaciones } \\
\text { congénitas debidas a } \\
\text { causas exógenas }\end{array}$ & 8 & 0,11 & 7 & 0,09 & 1 & 1,90 & 14,29 \\
\hline $\begin{array}{l}\text { Q873, } \\
\text { Q875y } \\
\text { Q878 }\end{array}$ & $\begin{array}{l}\text { Sd con exceso de } \\
\text { crecimiento precoz, con } \\
\text { otros cambios esqueléticos } \\
\text { o no clasificados }\end{array}$ & 7 & 0,09 & 6 & 0,08 & 1 & 1,90 & 16,67 \\
\hline Q761 & Síndrome de Klippel-Feil & 2 & 0,03 & 2 & 0,03 & 0 & - & - \\
\hline $\begin{array}{l}\text { Q920 y } \\
\text { Q927 }\end{array}$ & $\begin{array}{l}\text { Otras trisomías no } \\
\text { clasificadas }\end{array}$ & 2 & 0,03 & 2 & 0,03 & 0 & - & - \\
\hline Total & TOTAL & 1065 & 14,09 & 1025 & 13,69 & 37,00 & 70,18 & 3,61 \\
\hline
\end{tabular}

no son tan evidentes al nacimiento y se diagnostican luego en forma clínica o por otros métodos diagnósticos. La prevalencia de malformaciones congénitas en Costa Rica, durante el período en estudio, fue del 1,47\% (1,44-1,49), similar al $1,5 \%(1,46-1,54)$ reportado antes. ${ }^{11}$ La prevalencia total de polimalformados fue de $34,8(33,4-36,1)$ por cada 10000 nacidos, lo cual es similar a lo reportado por otros países: Cuba reportó 44,3 de polimalformados por cada 10 000 nacimientos, con una prevalencia de malformaciones al nacimiento del $1,7 \%$, el $24,6 \%$ de los malformados fueron polimalformados, ${ }^{1}$ porcentaje muy similar al encontrado en este análisis $(23,7 \%)$. Hungría reportó una prevalencia de polimalformados de 45,1 por 10000 nacimientos ${ }_{15}^{15} \mathrm{y}$ España, 48,8 por 10000 nacimientos; en este último país la prevalencia de malformaciones fue del $2 \%$, siendo el $25 \%$ de ellas malformados múltiples. ${ }^{16}$

En los óbitos, el porcentaje de distribución entre malformados simples y polimalformados varía, siendo significativamente mayor el porcentaje en los polimalformados. Se encontró que el porcentaje de polimalformados aumentó del $23,2 \%$ en nacidos vivos al $45,3 \%$ en óbitos, con una prevalencia 5,48 veces mayor que la de recién nacidos vivos. Existen varios estudios donde se 


\begin{tabular}{|c|c|c|c|c|c|c|}
\hline \multirow{2}{*}{ Provincia } & \multicolumn{3}{|c|}{$1996-2000$} & \multicolumn{3}{|c|}{$2001-2005$} \\
\hline & \# Casos & $\%$ polimalf. & IC 95\% & \# Casos & \% polimalf. & IC 95\% \\
\hline San José & 2081 & $23,0 \%$ & $(21,2-24,8)$ & 1863 & $23,3 \%$ & $(21,4-25,2)$ \\
\hline Alajuela & 695 & $27,5 \%$ & $(24,2-30,8)$ & 738 & $24,5 \%$ & $(21,4-27,6)$ \\
\hline Cartago & 778 & $18,9 \%$ & $(16,1-21,6)$ & 769 & $15,5 \%$ & $(12,9-18,0)$ \\
\hline Heredia & 713 & $18,0 \%$ & $(15,3-20,9)$ & 456 & $22,4 \%$ & $(18,5-26,2)$ \\
\hline Guanacaste & 231 & $29,8 \%$ & $(24,0-35,7)$ & 192 & $28,1 \%$ & $(21,8-34,5)$ \\
\hline Puntarenas & 459 & $29,4 \%$ & $(25,2-33,6)$ & 481 & $25,2 \%$ & $(21,3-29,0)$ \\
\hline Limón & 502 & $23,3 \%$ & $(19,6-27,0)$ & 766 & $29,1 \%$ & $(25,9-32,3)$ \\
\hline Sin datos & 98 & $23,5 \%$ & $(15,1-31,9)$ & 50 & $22,0 \%$ & $(10,5-33,5)$ \\
\hline Total & 5557 & $23,0 \%$ & $(21,9-24,2)$ & 5318 & $23,4 \%$ & $(22,3-24,5)$ \\
\hline
\end{tabular}

\begin{tabular}{|lcccc|}
\hline \multicolumn{3}{|c|}{$\begin{array}{c}\text { Cuadro 6. Prevalencia de recién nacidos con } \\
\text { malformaciones múltiples, según provincia de } \\
\text { residencia de la madre y año de nacimiento, } \\
\text { Costa Rica, 1996-2005 }\end{array}$} \\
\hline \multicolumn{4}{|c}{$1996-2000$} & \multicolumn{3}{c|}{$2001-2005$} \\
Provincia & Prevalencia* & IC 95\% & Prevalencia* & IC95\% \\
\hline San José & 35,7 & $(32,5-38,9)$ & 35,8 & $(32,4-38,9)$ \\
Alajuela & 25,9 & $(22,3-29,7)^{\text {a }}$ & 26,4 & $(22,6-30,1)^{\text {a }}$ \\
Cartago & 35,2 & $(29,5-40,9)$ & 32,2 & $(26,4-37,7)$ \\
Heredia & 39,1 & $(32,4-45,9)$ & 31,3 & $(25,3-37,4)$ \\
Guanacaste & 22,3 & $(16,6-27,9)^{a}$ & 20,9 & $(15,3-26,3)^{\text {a }}$ \\
Puntarenas & 33,1 & $(27,5-38,7)$ & 32,6 & $(26,8-38,2)$ \\
Limón & 28,6 & $(23,5-33,8)$ & 59,3 & $(51,6-66,7)^{\text {bc }}$ \\
Costa Rica & 32,8 & $(31,0-34,6)$ & 34,6 & $(32,7-36,4)$ \\
\hline
\end{tabular}

* Prevalencia por 10000 nacidos

a Prevalencia significativamente menor $(p<0.05)$ a la nacional en ese mismo período, $b$ Prevalencia significativamente mayor a la nacional en ese mismo período, c Prevalencia significativamente mayor con respecto al período anterior.

evidencia el aumento en el porcentaje de distribución de los polimalformados en los recién nacidos fallecidos. En un estudio en España este porcentaje aumentó del 25\% al 54\%, ${ }^{16}$ incremento similar al presentado en nuestro análisis (de 23,2 a $45,3 \%)$. Por otro lado, la distribución porcentual de los polimalformados en síndromes y malformaciones asociadas es similar a la presentada en otros países. En Costa Rica el $40,5 \%$ de los polimalformados corresponden a síndromes ya reconocidos y clasificados según CIE10; el 59,5\% restante corresponde a la asociación de 2 ó más malformaciones. En España se encontró que los porcentajes oscilaban en el $43,8 \%$ y el $56 \%$, respectivamente.
Al analizar el comportamiento en el tiempo de los polimalformados durante el período en estudio, se evidencia que hubo un aumento significativo de la prevalencia durante 2001. Después de este año, las prevalencias descienden a valores similares a los presentados durante el primer quinquenio. Este aumento se presentó en todos los casos, tanto simples como polimalformados. Se deben realizar análisis más profundos para identificar si ello se debe a mejoras en el registro durante ese año o si efectivamente se presentó una elevación de las prevalencias. Llama la atención, además, el aumento significativo durante el segundo quinquenio analizado, de la prevalencia de polimalformados en la provincia de Limón. Esta fue la única que presentó una prevalencia significativamente mayor a la presentada en el nivel nacional; se deben hacer estudios analíticos para indagar la causa de ello; por otro lado, las provincias de Alajuela y Guanacaste presentaron prevalencias menores a las nacionales, una posibilidad es que en ellas haya subregistro de la información.

Los hallazgos encontrados en la regresión logística no condicionada concuerdan con los resultados obtenidos en la descripción de las variables; la prevalencia de malformados y polimalformados varía según la edad de la madre, el sexo del recién nacido y la provincia de residencia. Las madres que tienen mayor riesgo de presentar un recién nacido malformado y polimalformado son las mayores de 35 años. El sexo del recién nacido que presenta un mayor riesgo de malformación es el masculino, y las provincias que presentaron una prevalencia menor que la esperada fueron las de Alajuela y Guanacaste.

Los resultados obtenidos al analizar las variables edad de la madre y sexo de niños son similares a los reportados en la bibliografía; es bien conocido que la edad materna avanzada constituye un factor de riesgo para parir un recién nacido con malformaciones, principalmente cromosomopatías. ${ }^{17}$ Ya está bien demostrado, por ejemplo, que mujeres mayores de 35 años tienen 4 veces más alto 
riesgo de tener hijos con Síndrome de Down, ${ }^{18} \mathrm{y}$ en nuestro análisis se demostró que las mujeres mayores de 35 años, tienen un riesgo de tener un polimalformado 2,4 veces mayor que las menores de 35 años. El riesgo de presentar una malformación simple también es mayor, pero en mucho menor grado que en los polimalformados.

Se ha documentado también que el sexo masculino constituye un factor de riesgo para tener malformaciones congénitas. ${ }^{19} \mathrm{La}$ prevalencia de polimalformados es significativamente mayor en el sexo masculino.

\section{Agradecimientos y colaboradores}

Al ingeniero Uber Calderón Alfaro, por su colaboración en el análisis estadístico y revisión del documento. Al Dr. Juan José Romero, por su asesoría en el diseño del protocolo de tesis.

\section{Referencias}

1. Alonso F, Cebdán I, Ferreo ME, Roca J, Castillo P, Petizco A et al. Caracterización patogénica de los recién nacidos con malformaciones múltiples. Rev Cubana Pediatr 1998; 70: 73-8.

2. Lynberg MC, Khoury MJ. Contribution of birth defect to infant mortality among racial/ethnic minority groups, United States, 1983. MMWR. 1990; 39:1-12.

3. Organización Panamericana de la Salud. La Salud de las América. Washington: OPS, 1998: 198-210.

4. Castro I. Pasado, presente y futuro de la citogenética en Costa Rica. Rev Biol Trop 2004; 52: 537-544.

5. Instituto Nacional de Estadística y Censos. Estadísticas vitales 2002, San José, Costa Rica: INEC, 2004: 81.

6. Cordero JF. Registro de defectos congénitos y enfermedades genéticas. Clínicas Pediátricas de Norteamérica. 1992; 1: 65-77.

\begin{tabular}{|c|c|c|c|c|c|c|c|}
\hline \multirow{3}{*}{$\begin{array}{l}\text { Grupo etáreo } \\
<\text { de } 20 \text { años }\end{array}$} & \multicolumn{3}{|c|}{ Malformado simple } & \multicolumn{2}{|c|}{ Malformado múltiple } & \multicolumn{2}{|c|}{ Total R.N malformados } \\
\hline & \multicolumn{2}{|c|}{ Prevalencia } & \multirow{2}{*}{$\frac{(I C 95 \%)}{6-116,5)}$} & \multicolumn{2}{|c|}{ Prevalencia (IC 95\%) } & \multicolumn{2}{|c|}{ Prevalencia (IC 95\%) } \\
\hline & 111,3 & $(10$ & & 30,4 & $(27,6-33,1)$ & 141,6 & $(135,7-147,6)$ \\
\hline 20 a 34 años & 108,6 & $(10$ & $5,8-111,5)$ & 28,9 & $(27,4-30,3)$ & 137,5 & $(134,3-143,4)$ \\
\hline$\geq 35$ años & 124,1 & $(11$ & $0,4-132)^{a}$ & 69,9 & $(64-75,7)^{a}$ & 194 & $(184-270,8)^{\mathrm{a}}$ \\
\hline
\end{tabular}

a Prevalencia significativamente mayor $(\mathrm{p}<0.05)$ a la presentada en el resto de los grupos etáreos

\begin{tabular}{|lcccccc|}
\hline \multicolumn{5}{|c|}{ Cuadro 8. Razones de prevalencia de recién nacidos con malformaciones, según número de } \\
malformaciones y edad de la madre, Costa Rica, 1996-2005
\end{tabular}

a Razón de prevalencia significativamente mayor a la presentada en malformados simples.

\begin{tabular}{|c|c|c|c|c|c|c|}
\hline \multicolumn{7}{|c|}{$\begin{array}{l}\text { Cuadro 9. Prevalencia de recién nacidos con malformaciones congénitas según sexo del recién } \\
\text { nacido, (prevalencia por } 10000 \text { nacidos), Costa Rica, 1996-2005 }\end{array}$} \\
\hline \multirow{2}{*}{$\begin{array}{c}\text { Sexo } \\
\text { Masculino }\end{array}$} & \multicolumn{2}{|c|}{$\begin{array}{l}\text { Malformado simple } \\
\text { Prevalencia } \quad \text { (IC 95\%) }\end{array}$} & \multicolumn{2}{|c|}{$\begin{array}{l}\text { Malformado múltiple } \\
\text { Prevalencia (IC 95\%) }\end{array}$} & \multicolumn{2}{|c|}{$\begin{array}{l}\text { Total R.N malformados } \\
\text { Prevalencia (IC 95\%) }\end{array}$} \\
\hline & 111,8 & $(108,5-115,2)$ & 33,6 & $(31,8-35,5)^{a}$ & 145,5 & $(141,7-149,3)^{a}$ \\
\hline Femenino & 106,6 & $(103,3-109,9)$ & 29,9 & $(28,2-31,7)$ & 136,6 & $(132,8-140,3)$ \\
\hline
\end{tabular}

a Prevalencia significativamente mayor a la presentada en el sexo femenino 
7. Lyons K. Dismorfología. En Behrman R, Kliegman R, Arvin A. Nelson tratado de pediatría. 15 ed. Madrid: McGraw-HillInteramericana, 1997: 593-596.

8. Martínez ML. Anomalía de Möebius y el concepto de secuencia malformativa: importancia del conocimiento y uso adecuado de la terminología. Boletín del ECEMC: Revista de Dismorfología y Epidemiología. 2003; 5: 10-14.

9. Ministerio de Salud. Boletín Epidemiológico. Sistema Nacional de Vigilancia de la Salud. Costa Rica: Ministerio Salud, 2002; 2:1-2.

10. Organización Panamericana de la Salud. Clasificación estadística internacional de enfermedades y problemas relacionados con la salud. CIE 10. Washington: OPS, 1995: 753-803.

11. INCIENSA. Centro de Registro de Enfermedades Congénitas CREC. Prevalencia de enfermedades congénitas por provincias y cantones 1987-2000. Tres Ríos, Costa Rica: INCIENSA. 2002.

12. INCIENSA. Centro de Registro de Enfermedades Congénitas (CREC). Manual para hospitales. INCIENSA. 1987.
13. Wayne D. Bioestadística: base para el análisis de las ciencias de la salud. México D.F.: Limusa Wiley, 2002: 176-178.

14. Thompson M, McInnes R, Willard H. Genetics in medicine. $4^{\mathrm{a}}$ ed. Filadelfia, Estados Unidos: WB Saunders, 1996: 8-11.

15. Czeizel A. A nation wide evaluation of múltiple congenital abnormalities in Hungary. Genet Epidemiol 1998; 5: 183-202.

16. Martínes ML, Salvador J, Adan A, Frías JL. Frecuencia de defectos congénitos. España 1976-1983. An Esp Pediatr 1986; 25: 145-53.

17. Cortés F. Prevención primaria de los defectos de cierre del tubo neural. Rev Chil Pediatr 2003; 74: 208-212.

18. Romero S, Quesada C, Barrera M, Cabrales L. Efecto de riesgo en el hijo de madre con edad avanzada (estudio de casos y controles). Ginecol Obstet Mex 2002; 70: 295-302.

19. Sacsaquispe S, Ortiz L. Prevalencia de labio y/o paladar fisurado y factores de riesgo. Rev Estomatol Herediana 2004; 14: 54-58.

\begin{tabular}{|c|c|c|c|c|c|c|}
\hline Variables & Coeficiente & $\begin{array}{c}\text { Error } \\
\text { estándar }\end{array}$ & Valor $\mathrm{p}$ & $\begin{array}{l}\text { Razón de } \\
\text { posibilidades }\end{array}$ & \multicolumn{2}{|c|}{ IC $95 \%$} \\
\hline$\% G M$ & $-4,4669$ & 0,0352 & $<0,001$ & 0,0115 & $(0,0107$ & $-0,0123)$ \\
\hline Edad $<20={ }^{\prime} 1^{\prime}$ & 0,0170 & 0,0251 & 0,4988 & 1,0171 & $(0,9683$ & $-1,0684)$ \\
\hline Edad 35 y más ='2' & 0,3019 & 0,0288 & $<0,001$ & 1,3524 & $(1,2783$ & $-1,4308)$ \\
\hline Sexo masculino =' 1 ' & 0,0691 & 0,0196 & $<0,001$ & 1,0715 & $(1,0311$ & $-1,1135)$ \\
\hline Alajuela =' 1 ' & $-0,4130$ & 0,0585 & $<0,001$ & 0,6617 & $(0,5900$ & $-0,7421)$ \\
\hline Cartago ='2' & 0,3697 & 0,0442 & $<0,001$ & 1,4473 & $(1,3271$ & $-1,5784)$ \\
\hline Heredia ='3' & 0,4796 & 0,0417 & $<0,001$ & 1,6155 & $(1,4888$ & $-1,7529)$ \\
\hline Guanacaste =' 4 ' & $-0,1974$ & 0,0422 & $<0,001$ & 0,8209 & $(0,7557$ & $-0,8917)$ \\
\hline Puntarenas =' 5 ' & 0,2785 & 0,0365 & $<0,001$ & 1,3212 & $(1,2299$ & $-1,4192)$ \\
\hline Limón ='6' & 0,2820 & 0,0433 & $<0,001$ & 1,3257 & $(1,2178$ & $-1,4432)$ \\
\hline
\end{tabular}

Deviance 68,74 con 32 grados de libertad, likelihood ratio test $9,0 \mathrm{E}+05$, con un valor de $p<0,001$. 